\title{
A path integral approach to noncommutative superspace
}

\author{
Iouri Chepelev and Calin Ciocarlie \\ Department of Physics, California Institute of Technology \\ Pasadena, CA 91125, USA \\ E-mail: chepelev@theory.caltech.edu, calin@theory.caltech.edu
}

\begin{abstract}
A path integral formula for the associative star-product of two superfields is proposed. It is a generalization of the Kontsevich-Cattaneo-Felder's formula for the starproduct of functions of bosonic coordinates. The associativity of the star-product imposes certain conditions on the background of our sigma model. For generic background the action is not supersymmetric. The supersymmetry invariance of the action constrains the background and leads to a simple formula for the star-product.
\end{abstract}

KEywords: Sigma Models, Topological Field Theories, Superstrings and Heterotic Strings, BRST Symmetry. 


\section{Contents}

1. Introduction 1

2. Path integral formula 2

3. A special case: supersymmetric backgrounds 7

4. Summary 10

\section{Introduction}

An outstanding problem is the following: Given a classical structure find its quantum extension(s). Deformation quantization theory is an interesting approach to this problem 国.

In [2, 3] it was shown that any symplectic manifold can be deformation-quantized. The question whether any Poisson manifold can be quantized was answered in affirmative only recently by Kontsevich 沟. The solution presented by Kontsevich uses in a essential way ideas of perturbative string theory. Kontsevich's construction was further clarified by Cattaneo and Felder in 5 , where an explicit path integral formula for the star-product of functions on the Poisson manifolds was given.

Unlike the star-product on the Grassmann-even manifolds, the star-product on the supermanifolds is not sufficiently studied. For a list of papers on the subject, see [0 7 . Ref. [7] gives a systematic analysis of the deformation of the algebra of four dimensional supercoordinates. ${ }^{1}$ The most general deformed $N=1, d=4$ supercoordinate algebra which satisfies Jacobi identities and is compatible with the $N=1$ supersymmetry reads

$$
\left[x^{i}, x^{j}\right]=i \hbar \mathbb{B}^{i j}(\theta), \quad\left[x^{i}, \theta^{\alpha}\right]=i \hbar \mathbb{C}^{\alpha i}, \quad\left\{\theta^{\alpha}, \theta^{\beta}\right\}=0,
$$

where $\mathbb{B}$ is $x$-independent and is a linear function of $\theta$. $\mathbb{C}$ is independent of $x$ and $\theta$, and satisfies an equation following from the Jacobi identity.

Recently, using Berkovits' covariant formalism for quantizing type-II superstring compactified on a Calabi-Yau three-fold [9], Ooguri and Vafa made an interesting observation that the RR field strengths can give rise to a non-zero $\{\theta, \theta\}$ anticommutator in four dimensions [10]. ${ }^{2}$ De Boer, Grassi and van Nieuwenhuizen [12] generalized this observation to ten dimensional superspace using Berkovits' formalism [13]. Ref. [12] also pointed out that the gravitino yields a non-zero value for $[x, \theta]$.

\footnotetext{
${ }^{1}$ For an earlier discussion of the quantum deformations of the Poincare supergroup, see 8 .

${ }^{2}$ For an earlier discussion of RR backgrounds in the context of noncommutativity in string theory, see 11.
} 
The purpose of this paper is to give a general path integral formula for the star-product of functions of $x$ and $\theta$. The $\star$-algebra which arises from our path integral formula is of the form

$$
\begin{aligned}
& {\left[x^{i}, x^{j}\right]_{\star}=i \hbar \mathbb{B}^{i j}(x, \theta)+O\left(\hbar^{2}\right),} \\
& {\left[x^{i}, \theta^{\alpha}\right]_{\star}=i \hbar \mathbb{C}^{\alpha i}(x, \theta)+O\left(\hbar^{2}\right), \quad\left\{\theta^{\alpha}, \theta^{\beta}\right\}_{\star}=i \hbar \mathbb{F}^{\alpha \beta}(x, \theta)+O\left(\hbar^{2}\right) .}
\end{aligned}
$$

Using path integral methods, we will derive the associativity equations satisfied by the fields $\mathbb{B}, \mathbb{C}$ and $\mathbb{F}$.

As a main tool in deriving the path integral formula, we use an elegant supermanifold formalism for the Batalin-Vilkovisky(BV) quantization [14] introduced by Alexandrov, Kontsevich, Schwarz and Zaboronsky [15]. This formalism was further elaborated and extended by Park [16]. ${ }^{3}$ The AKSZ method [15] is a method to construct solutions of the BV master equation directly, without starting from a classical action with a set of symmetries, as is done in the BV method. The classical action is then recovered a posteriori by setting the fields of non-zero ghost number to zero. In ten spacetime dimensions the classical action that we obtain from our BV-action using this method will turn out to be equivalent, for the constant background fields, to the one used in [12].

The star-product will be represented as a path integral of a sigma model on a superdisk. The BV-action $S=S[\boldsymbol{X}, \boldsymbol{\Theta}, \boldsymbol{\rho}, \boldsymbol{\xi}]$ of the sigma model will be a functional of the superspacevalued superfields. The action $S$ depends on the background fields $\mathbb{B}, \mathbb{C}$ and $\mathbb{F}$. Imposing the supersymmetry invariance of the action, we will obtain the algebra (1.1). We will also find an explicit formula for the supersymmetric star-product of functions of $x$ and $\theta$.

The paper is organized as follows. In section 2 the path integral formula is given, the associativity equations are derived and the Feynman diagrammatics is presented. The supersymmetric backgrounds are considered in section 3 where an explicit expression for the star-product is given. A summary of our results can be found in section 4 .

\section{Path integral formula}

The star-product is represented as a path integral on a superdisk with the two functions inserted on the boundary of the disk:

$$
\begin{aligned}
f \star g(x, \theta)=\int_{X(\infty)=x, \Theta(\infty)=\theta} & f(X(0), \Theta(0)) g(X(1), \Theta(1)) \times \\
& \times\left.\mathrm{e}^{\frac{i}{\hbar} S[\boldsymbol{X}, \boldsymbol{\Theta}, \boldsymbol{\rho}, \boldsymbol{\xi}]} D \boldsymbol{X} D \boldsymbol{\Theta} D \boldsymbol{\rho} D \boldsymbol{\xi}\right|_{\substack{\text { lagrangian } \\
\text { submanifold }}}
\end{aligned}
$$

where 0 and 1 are the points on the boundary of the disk where the functions are inserted. The path integral is computed with the condition $X=x, \Theta=\theta$ at the point $\infty$ of the boundary of the disk. The path integral is taken over a lagrangian submanifold in the field space $\{\boldsymbol{X}, \boldsymbol{\Theta}, \boldsymbol{\rho}, \boldsymbol{\xi}\}$.

\footnotetext{
${ }^{3}$ See also 17 .
} 
We first describe the fields that appear in eq. (2.1). $\boldsymbol{X}, \boldsymbol{\Theta}, \boldsymbol{\rho}$ and $\boldsymbol{\xi}$ are maps from the superdisk to a superspace $\mathcal{M}$. The local coordinates of $\mathcal{M}$ are $X^{i}, \Theta^{\alpha}, \rho_{i}$ and $\xi_{\alpha}$. For simplicity we work in the spacetime dimension $n$ that admits a Majorana representation. $\Theta$ is a Majorana spinor in $n$ dimensions. $i$ is a spacetime index and $\alpha$ is a spinorial index. The coordinates of $\mathcal{M}$ are graded with respect to two Grassmann gradations $\varepsilon_{1}$ and $\varepsilon_{2}$ :

$$
\begin{array}{llll}
\varepsilon_{1}(X)=0, & \varepsilon_{1}(\Theta)=1, & \varepsilon_{1}(\rho)=0, & \varepsilon_{1}(\xi)=1, \\
\varepsilon_{2}(X)=0, & \varepsilon_{2}(\Theta)=0, & \varepsilon_{2}(\rho)=1, & \varepsilon_{2}(\xi)=1 .
\end{array}
$$

The commutation property of functions on $\mathcal{M}$ is given by the relation

$$
f g=(-1)^{\varepsilon_{1}(f) \varepsilon_{1}(g)+\varepsilon_{2}(f) \varepsilon_{2}(g)} g f .
$$

Let $\sigma^{\mu}$ and $\zeta^{\mu}(\mu=1,2)$ be bosonic and fermionic coordinates of the superdisk. Then

$$
\boldsymbol{X}=\boldsymbol{X}(\sigma, \zeta), \quad \boldsymbol{\Theta}=\boldsymbol{\Theta}(\sigma, \zeta), \quad \boldsymbol{\rho}=\boldsymbol{\rho}(\sigma, \zeta), \quad \boldsymbol{\xi}=\boldsymbol{\xi}(\sigma, \zeta) .
$$

In other words we have an embedding $\phi$ of the superdisk $D$ into the superspace $\mathcal{M}$ :

$$
\phi: D \rightarrow \mathcal{M} .
$$

We now describe the action $S$ in eq. (2.1). It is an integral over the superdisk $D$

$$
S=S_{0}+S_{\text {int }}=\int_{D} d^{2} \sigma d^{2} \zeta\left(\mathcal{L}_{0}+\mathcal{H}\right)=\int_{D} d^{2} \sigma d^{2} \zeta \mathcal{L}
$$

of the lagrangian density

$$
\begin{aligned}
\mathcal{L} & =\mathcal{L}_{0}+\mathcal{H} \\
\mathcal{L}_{0} & =\boldsymbol{\rho}_{i} D \boldsymbol{X}^{i}+\boldsymbol{\xi}_{\alpha} D \boldsymbol{\Theta}^{\alpha} \\
\mathcal{H} & =\frac{1}{2} \mathbb{B}^{i j}(\boldsymbol{X}, \boldsymbol{\Theta}) \boldsymbol{\rho}_{i} \boldsymbol{\rho}_{j}+\frac{1}{2} \mathbb{F}^{\alpha \beta}(\boldsymbol{X}, \boldsymbol{\Theta}) \boldsymbol{\xi}_{\alpha} \boldsymbol{\xi}_{\beta}+\mathbb{C}^{\alpha i}(\boldsymbol{X}, \boldsymbol{\Theta}) \boldsymbol{\xi}_{\alpha} \boldsymbol{\rho}_{i} .
\end{aligned}
$$

$D$ in eq. (2.7) is defined as

$$
D=\zeta^{\mu} \frac{\partial}{\partial \sigma^{\mu}}
$$

The action $S$ is a Batalin-Vilkovisky (BV) action. The components of the superfields $\boldsymbol{X}$, $\boldsymbol{\Theta}, \boldsymbol{\rho}$ and $\boldsymbol{\xi}$ in the expansion in $\zeta$,

$$
\begin{aligned}
\boldsymbol{\xi} & =\xi^{(0)}+\xi_{\mu}^{(1)} \zeta^{\mu}+\xi_{\mu \nu}^{(2)} \zeta^{\mu} \zeta^{\nu}, \\
\boldsymbol{\rho} & =\rho^{(0)}+\rho_{\mu}^{(1)} \zeta^{\mu}+\rho_{\mu \nu}^{(2)} \zeta^{\mu} \zeta^{\nu}, \\
\boldsymbol{\Theta} & =\Theta^{(0)}+\Theta_{\mu}^{(1)} \zeta^{\mu}+\Theta_{\mu \nu}^{(2)} \zeta^{\mu} \zeta^{\nu}, \\
\boldsymbol{X} & =X^{(0)}+X_{\mu}^{(1)} \zeta^{\mu}+X_{\mu \nu}^{(2)} \zeta^{\mu} \zeta^{\nu},
\end{aligned}
$$

can be identified with the fields and antifields in the BV quantization of a classical action $S_{c l}$. The latter can be obtained from eq. (2.6) setting to zero fields with non-zero ghost number. The ghost numbers of the component fields in eq. (2.9) can be computed by 
assigning ghost number one to $\zeta$ and taking overall ghost number of the superfield to be given by the parity $\varepsilon_{2}$ defined in eq. (2.2). Thus we find

$$
\operatorname{gh}\left(X^{(k)}\right)=-k, \quad \operatorname{gh}\left(\Theta^{(k)}\right)=-k, \quad \operatorname{gh}\left(\rho^{(k)}\right)=1-k, \quad \operatorname{gh}\left(\xi^{(k)}\right)=1-k, \quad k=0,1,2 .
$$

Note that $\mathcal{H}$ is the most general ghost number two interaction term that can be constructed out of $\boldsymbol{X}, \boldsymbol{\Theta}, \boldsymbol{\rho}$ and $\boldsymbol{\xi}$. Setting to zero fields with a non-zero ghost number in eq. (2.6), we find

$$
\begin{aligned}
S_{c l}=\int[ & \rho_{i}^{(1)} d X^{(0) i}+\xi_{\alpha}^{(0)} d \Theta^{(0) \alpha}+\frac{1}{2} \mathbb{B}^{i j}\left(X^{(0)}, \Theta^{(0)}\right) \rho_{i}^{(1)} \rho_{j}^{(1)}+ \\
& \left.+\frac{1}{2} \mathbb{F}^{\alpha \beta}\left(X^{(0)}, \Theta^{(0)}\right) \xi_{\alpha}^{(1)} \xi_{\beta}^{(1)}+\mathbb{C}^{\alpha i}\left(X^{(0)}, \Theta^{(0)}\right) \xi_{\alpha}^{(1)} \rho_{i}^{(1)}\right],
\end{aligned}
$$

where

$$
\rho_{i}^{(1)}=\rho_{i \mu}^{(1)} d \sigma^{\mu}, \quad \xi_{\alpha}^{(1)}=\xi_{\alpha \mu}^{(1)} d \sigma^{\mu}
$$

are one forms. ${ }^{4}$

In the BV quantization the action $S$ must satisfy the master equation [14]:

$$
(S, S)_{\mathrm{BV}}-2 i \hbar \Delta S=0
$$

where $(\cdot, \cdot)_{\mathrm{BV}}$ is the BV-bracket and $\Delta$ is the BV-laplacian. It can be checked that [5]

$$
\Delta S=0
$$

Thus the master equation becomes

$$
(S, S)_{\mathrm{BV}}=0
$$

The associativity of the star-product follows from the Ward identity as in [0]. Thus eq. (2.15) implies the associativity of the star-product:

$$
(S, S)_{\mathrm{BV}}=0 \quad \Longrightarrow \quad \text { the star-product is associative }
$$

We decompose the action $S$ into the kinetic $S_{0}$ and the interaction $S_{\text {int }}$ parts as in eq. (2.6). It is easy to show that (see [16]):

$$
\left(S_{0}, \boldsymbol{X}\right)_{\mathrm{BV}}=D \boldsymbol{\rho}, \quad\left(S_{0}, \boldsymbol{\rho}\right)_{\mathrm{BV}}=D \boldsymbol{X}, \quad\left(S_{0}, \boldsymbol{\Theta}\right)_{\mathrm{BV}}=D \boldsymbol{\xi}, \quad\left(S_{0}, \boldsymbol{\xi}\right)_{\mathrm{BV}}=D \boldsymbol{\Theta} .
$$

Thus we have

$$
\left(S_{0}, S_{\mathrm{int}}\right)_{\mathrm{BV}}=\int_{D} D \mathcal{H}
$$

\footnotetext{
${ }^{4}$ Integrating out $\rho^{(1)}$ in $S_{c l}$, we end up with an action which is, for constant background fields, equivalent to the action (without the $G \partial X \partial X$ term) used in [12]. To be more precise, the two actions are equivalent modulo the difference in the type of spinors considered: we have type-IIA spinors whereas ref. [12] used type-IIB.
} 
Since the integrand on the r.h.s. of this equation is a total derivative, the integral can be reduced to an integral over the boundary of the disk. This boundary integral vanishes due to the boundary conditions for the superfields:

$$
\begin{aligned}
& X_{\mu}^{(1)} n^{\mu}=0, \quad X_{\mu \nu}^{(2)}=0, \quad \rho^{(0)}=0, \quad \rho_{\mu}^{(1)} t^{\mu}=0, \\
& \Theta_{\mu}^{(1)} n^{\mu}=0, \quad \Theta_{\mu \nu}^{(2)}=0, \quad \xi^{(0)}=0, \quad \xi_{\mu}^{(1)} t^{\mu}=0,
\end{aligned}
$$

where $n^{\mu}$ and $t^{\mu}$ are normal and tangential vectors to the boundary of the disk. ${ }^{5}$ Thus we have $\left(S_{0}, S_{\mathrm{int}}\right)_{\mathrm{BV}}=0$. Similarly, we can show that $\left(S_{0}, S_{0}\right)_{\mathrm{BV}}=0$. Thus eq. (2.15) reduces to

$$
\left(S_{\text {int }}, S_{\text {int }}\right)_{\mathrm{BV}}=0 .
$$

Eq. (2.20) implies that the background fields $\mathbb{B}, \mathbb{F}$ and $\mathbb{C}$ satisfy certain equations. We now derive these equations. The calculations can be significantly simplified if we use the following formula [15, 16] which relates the BV-bracket to a bracket $[\cdot, \cdot]$ on the superspace $\mathcal{M}$ :

$$
\int_{D} \phi^{*}([f, g])=\left(\int_{D} \phi^{*} f, \int_{D} \phi^{*} g\right)_{\mathrm{BV}} .
$$

Here $\phi^{*} f$ and $\phi^{*} g$ are pullbacks of the functions $f$ and $g$ on $\mathcal{M}$ to the superdisk $D$ with respect to the map $\phi$ from eq. (2.5). The bracket $[f, g]$ is given by

$$
[f, g]=\frac{\partial_{r} f}{\partial \rho_{i}} \frac{\partial g}{\partial X^{i}}-\frac{\partial f}{\partial X^{i}} \frac{\partial_{l} g}{\partial \rho_{i}}+\frac{\partial_{r} f}{\partial \xi_{\alpha}} \frac{\partial_{l} g}{\partial \Theta^{\alpha}}+\frac{\partial_{r} f}{\partial \Theta^{\alpha}} \frac{\partial_{l} g}{\partial \xi_{\alpha}} .
$$

where $r$ and $l$ denote the right and the left partial derivatives. ${ }^{6}$ Using eq. (2.21), eq. (2.20) is equivalent to

$$
[\mathcal{H}, \mathcal{H}]=0,
$$

where $\mathcal{H}$ is a function on $\mathcal{M}$ :

$$
\mathcal{H}=\frac{1}{2} \mathbb{B}^{i j}(X, \Theta) \rho_{i} \rho_{j}+\frac{1}{2} \mathbb{F}^{\alpha \beta}(X, \Theta) \xi_{\alpha} \xi_{\beta}+\mathbb{C}^{\alpha i}(X, \Theta) \xi_{\alpha} \rho_{i} .
$$

Since $\varepsilon_{1}(\mathcal{H})=\varepsilon_{2}(\mathcal{H})=0$, we have

$$
[\mathcal{H}, \mathcal{H}]=2\left(\frac{\partial_{r} \mathcal{H}}{\partial \rho_{i}} \frac{\partial \mathcal{H}}{\partial X^{i}}+\frac{\partial \mathcal{H}}{\partial \xi_{\alpha}} \frac{\partial_{l} \mathcal{H}}{\partial \Theta^{\alpha}}\right) .
$$

From the equations (2.23), (2.24) and eq. (2.25) we find

$$
\begin{aligned}
\left(\mathbb{C}^{\alpha i} \partial_{\alpha} \mathbb{B}^{j k}+\mathbb{B}^{i l} \partial_{l} \mathbb{B}^{j k}\right) \rho_{i} \rho_{j} \rho_{k} & =0, \\
\left(\mathbb{F}^{\alpha \delta} \partial_{\delta} \mathbb{F}^{\beta \gamma}+\mathbb{C}^{\alpha i} \partial_{i} \mathbb{F}^{\beta \gamma}\right) \xi_{\alpha} \xi_{\beta} \xi_{\gamma} & =0, \\
\left(\mathbb{F}^{\alpha \beta} \partial_{\beta} \mathbb{B}^{i j}-2 \mathbb{C}^{\beta i} \partial_{\beta} \mathbb{C}^{\alpha j}-2 \mathbb{B}^{i k} \partial_{k} \mathbb{C}^{\alpha j}+\mathbb{C}^{\alpha k} \partial_{k} \mathbb{B}^{i j}\right) \xi_{\alpha} \rho_{i} \rho_{j} & =0, \\
\left(2 \mathbb{F}^{\alpha \delta} \partial_{\delta} \mathbb{C}^{\beta k}-\mathbb{C}^{\delta k} \partial_{\delta} \mathbb{F}^{\alpha \beta}+2 \mathbb{C}^{\alpha i} \partial_{i} \mathbb{C}^{\beta k}-\mathbb{B}^{k j} \partial_{j} \mathbb{F}^{\alpha \beta}\right) \xi_{\alpha} \xi_{\beta} \rho_{k} & =0,
\end{aligned}
$$

\footnotetext{
${ }^{5}$ The components $X^{(0)}$ and $\Theta^{(0)}$ satisfy the conditions: $X^{(0)}(\infty)=x$ and $\Theta^{(0)}(\infty)=\theta$.

${ }^{6}$ Eq. 2.22) follows from a general formula for the (odd) Poisson bracket on the supermanifold $\mathcal{M}$ [15. Let $z^{a}$ be the local coordinates of $\mathcal{M}$ and let $\omega=d z^{a} \omega_{a b} d z^{b}$ be an odd non-degenerate closed 2-form on $\mathcal{M}$. In our case it is given by $\omega=2\left(d X^{i} \wedge d \rho_{i}+d \Theta^{\alpha} \wedge d \xi_{\alpha}\right)$. The Poisson bracket is given by $[f, g]=\frac{\partial_{r} f}{\partial z^{a}} \omega^{a b} \frac{\partial_{l} g}{\partial z^{b}}$, where $\omega^{a b}$ is the inverse matrix of $\omega_{a b}$.
} 
where $\partial_{i}=\partial / \partial X^{i}$ and $\partial_{\alpha}=\partial_{l} / \partial \Theta^{\alpha}$. Eq. (2.26) is a generalization of the familiar Jacobi identity for the Poisson bivector $B^{i j}$ :

$$
B^{i l} \partial_{l} B^{j k}+B^{j l} \partial_{l} B^{k i}+B^{k l} \partial_{l} B^{i j}=0 .
$$

We now explain the last ingredient of the formula (2.1): the integration over a lagrangian submanifold in the field space $\{\boldsymbol{X}, \boldsymbol{\Theta}, \boldsymbol{\rho}, \boldsymbol{\xi}\}$. A final step in the BV quantization procedure involves choosing a ghost number -1 function $\Psi$, the "gauge fixing fermion", of the fields [14]. The path integral is then taken over the lagrangian submanifold defined by the equations:

$$
\text { antifield }=\frac{\partial \Psi}{\partial \text { field }} .
$$

We refer the reader to ref. [5] for the details on how new fields, called antighosts and Lagrange multipliers together with their antifields, are added to the set of fields $\boldsymbol{X}$ and $\boldsymbol{\rho}$. In a very similar way, we can add antighosts and Lagrange multipliers together with their antifields to $\Theta$ and $\boldsymbol{\xi}$. This procedure results in the following operational formula for the star-product:

$$
\begin{aligned}
f \star g(x, \theta)=\langle & f(x+\boldsymbol{X}(0,0), \theta+\boldsymbol{\Theta}(0,0)) \times \\
& \left.\times \mathrm{e}^{\frac{i}{\hbar} S_{\mathrm{int}}[x+\boldsymbol{X}, \theta+\boldsymbol{\Theta}, \boldsymbol{\rho}, \boldsymbol{\xi}]} g(x+\boldsymbol{X}(1,0), \theta+\boldsymbol{\Theta}(1,0))\right\rangle,
\end{aligned}
$$

where $S_{\text {int }}$ is given by eq. (2.6) and eq. (2.7). ${ }^{7}$ The Wick-contraction $\langle\cdots\rangle$ in eq. (2.29) is done using the super-propagators:

$$
\begin{aligned}
\left\langle\boldsymbol{X}^{k}\left(z_{1}, \zeta_{1}\right) \boldsymbol{\rho}_{j}\left(z_{2}, \zeta_{2}\right)\right\rangle & =\frac{i \hbar}{2 \pi} \delta_{j}^{k} \mathcal{D} \phi\left(z_{2}, z_{1}\right), \\
\left\langle\boldsymbol{\Theta}^{\alpha}\left(z_{1}, \zeta_{1}\right) \boldsymbol{\xi}_{\beta}\left(z_{2}, \zeta_{2}\right)\right\rangle & =\frac{i \hbar}{2 \pi} \delta_{\beta}^{\alpha} \mathcal{D} \phi\left(z_{2}, z_{1}\right),
\end{aligned}
$$

where

$$
\phi(z, w)=\frac{1}{2 i} \ln \frac{(z-w)(z-\bar{w})}{(\bar{z}-\bar{w})(\bar{z}-w)}
$$

and

$$
\mathcal{D}=\zeta_{1}^{\mu} \frac{\partial}{\partial z_{1}^{\mu}}+\zeta_{2}^{\mu} \frac{\partial}{\partial z_{2}^{\mu}} .
$$

Note that points $z_{1}$ and $z_{2}$ from eq. (2.30) belong to the upper-half complex plane. A generic term in the perturbative expansion in eq. (2.29) is of the form

$$
\begin{aligned}
\int \prod d z_{j} d \zeta_{j} \prod d z_{j}^{\prime} d \zeta_{j}^{\prime}\langle & \boldsymbol{X}\left(z_{1}, \zeta_{1}\right) \cdots \boldsymbol{X}\left(z_{N}, \zeta_{N}\right) \boldsymbol{\rho}\left(z_{N+1}, \zeta_{N+1}\right) \cdots \boldsymbol{\rho}\left(z_{2 N}, \zeta_{2 N}\right) \times \\
& \left.\times \boldsymbol{\Theta}\left(z_{1}^{\prime}, \zeta_{1}^{\prime}\right) \cdots \boldsymbol{\Theta}\left(z_{M}^{\prime}, \zeta_{M}^{\prime}\right) \boldsymbol{\xi}\left(z_{M+1}^{\prime}, \zeta_{M+1}^{\prime}\right) \cdots \boldsymbol{\xi}\left(z_{2 M}^{\prime}, \zeta_{2 M}^{\prime}\right)\right\rangle
\end{aligned}
$$

\footnotetext{
${ }^{7}$ The tadpole diagrams in eq. (2.29) are discarded. This can be achieved by the introduction of terms linear in $\boldsymbol{\rho}$ and $\boldsymbol{\xi}$ in the action: $\partial_{i} \mathbb{B}^{i j} \boldsymbol{\rho}_{j}, \partial_{\alpha} \mathbb{F}^{\alpha \beta} \boldsymbol{\xi}_{\beta}, \partial_{\alpha} \mathbb{C}^{\alpha i} \boldsymbol{\rho}_{i}$ and $\partial_{i} \mathbb{C}^{\alpha i} \boldsymbol{\xi}_{\alpha}$.
} 
times an expression involving partial derivatives of $f(x, \theta), g(x, \theta), \mathbb{B}(x, \theta), \mathbb{F}(x, \theta)$ and $\mathbb{C}(x, \theta)$. Wick-contracting using eq. (2.30) and integrating over the Grassmann variables $\zeta$ 's in eq. (2.33), we end up with a sum of expressions of the form

$$
\int d \phi\left(z_{i_{1}}, z_{j_{1}}\right) \wedge d \phi\left(z_{i_{2}}, z_{j_{2}}\right) \cdots d \phi\left(z_{i_{N}}, z_{j_{N}}\right) \wedge d \phi\left(z_{i_{1}}^{\prime}, z_{j_{1}}^{\prime}\right) \wedge d \phi\left(z_{i_{2}}^{\prime}, z_{j_{2}}^{\prime}\right) \cdots d \phi\left(z_{i_{M}}^{\prime}, z_{j_{M}}^{\prime}\right)
$$

where $d \phi$ is defined as

$$
d \phi(z, w)=d z \frac{\partial}{\partial z} \phi(z, w)+d w \frac{\partial}{\partial w} \phi(z, w) .
$$

\section{A special case: supersymmetric backgrounds}

The kinetic part $S_{0}$ of the action (2.6) is invariant under the following supersymmetry transformation:

$$
\begin{aligned}
\delta \boldsymbol{\Theta}^{\alpha} & =\epsilon^{\alpha}, \\
\delta \boldsymbol{X}^{k} & =i \bar{\epsilon} \Gamma^{k} \boldsymbol{\Theta}, \\
\delta \boldsymbol{\xi}_{\alpha} & =-i \boldsymbol{\rho}_{k} \bar{\epsilon}^{\beta} \Gamma_{\beta \alpha}^{k}, \\
\delta \boldsymbol{\rho}_{k} & =0 .
\end{aligned}
$$

Since $\Theta$ is a Majorana spinor, the transformation (3.1) is an $N=1$ or $N=2$ supersymmetry transformation depending on the dimension $n$ of the spacetime. For $n=3,4,8,9$ it is $N=1$ and for $n=2,10$ it is $N=2$. Imposing the invariance of the full action (2.6) under the transformation (3.1), we find that the background fields are further constrained. We will shortly show that the associativity and supersymmetry constrain the background fields as follows:

$$
\mathbb{F}=0
$$

$\mathbb{B}$ is $X$-independent and is linear in $\Theta$ :

$$
\mathbb{B}^{i j}(\Theta)=B^{i j}+i \Theta^{\alpha}\left[\left(\Gamma^{0} \Gamma^{i}\right)_{\alpha \beta} \mathbb{C}^{\beta j}-\left(\Gamma^{0} \Gamma^{j}\right)_{\alpha \beta} \mathbb{C}^{\beta i}\right]
$$

$\mathbb{C}$ is constant and satisfies the following equation

$$
\sum_{\pi \in \text { permutations of } i, j, k}(-1)^{\pi} \mathbb{C}^{\alpha \pi(i)}\left(\Gamma^{0} \Gamma^{\pi(j)}\right)_{\alpha \beta} \mathbb{C}^{\beta \pi(k)}=0 .
$$

Thus we recover the result of [7] on the deformation of $N=1, d=4$ supercoordinate algebra. In our calculations we do not assume that the spacetime dimension is four. Thus the form of the deformed supercoordinate algebra that we get from our star-product is the same in any spacetime dimension that admits a Majorana representation.

In the supersymmetric case we consider $x$-independent backgrounds. The action (2.6) is supersymmetric if the background fields satisfy the following equations

$$
\begin{aligned}
\partial_{\gamma} \mathbb{F}^{\alpha \beta} \xi_{\alpha} \xi_{\beta} & =0, \\
\left(\partial_{\gamma} \mathbb{C}^{\beta j}+i\left(\Gamma^{0} \Gamma^{j}\right)_{\gamma \alpha} \mathbb{F}^{\alpha \beta}\right) \xi_{\beta} \rho_{j} & =0, \\
\left(\partial_{\alpha} \mathbb{B}^{i j}-2 i\left(\Gamma^{0} \Gamma^{i}\right)_{\alpha \beta} \mathbb{C}^{\beta j}\right) \rho_{i} \rho_{j} & =0 .
\end{aligned}
$$


The first equation in eq. (3.5) implies that $\mathbb{F}^{\alpha \beta}$ does not depend on $\Theta$. For the $x$ independent backgrounds and the constant $\mathbb{F}$ the master equation (2.26) reduces to

$$
\begin{aligned}
\mathbb{C}^{\alpha i} \partial_{\alpha} \mathbb{B}^{j k} \rho_{i} \rho_{j} \rho_{k} & =0, \\
\left(\mathbb{F}^{\alpha \beta} \partial_{\beta} \mathbb{B}^{i j}-2 \mathbb{C}^{\beta i} \partial_{\beta} \mathbb{C}^{\alpha j}\right) \xi_{\alpha} \rho_{i} \rho_{j} & =0, \\
\mathbb{F}^{\alpha \delta} \partial_{\delta} \mathbb{C}^{\beta k} \xi_{\alpha} \xi_{\beta} \rho_{k} & =0 .
\end{aligned}
$$

Since $\xi$ 's commute, the third equation in eq. (3.6) is equivalent to

$$
\mathbb{F}^{\alpha \delta} \partial_{\delta} \mathbb{C}^{\beta k}+\mathbb{F}^{\beta \delta} \partial_{\delta} \mathbb{C}^{\alpha k}=0 .
$$

Using the second equation from eq. (3.5), eq. (3.7) can be rewritten as

$$
\mathbb{F}^{\alpha \gamma}\left(\Gamma^{0} \Gamma^{k}\right)_{\gamma \delta} \mathbb{F}^{\delta \beta}+\mathbb{F}^{\beta \gamma}\left(\Gamma^{0} \Gamma^{k}\right)_{\gamma \delta} \mathbb{F}^{\delta \alpha}=0 .
$$

Setting $k=0$ in this equation, we have

$$
\mathbb{F}^{\alpha \mu} \delta_{\mu \nu} \mathbb{F}^{\nu \beta}=0
$$

Since the action $(2.6)$ is hermitian, $\mathbb{F}$ is purely imaginary. Furthermore, $\mathbb{F}$ is symmetric: $\mathbb{F}^{\alpha \beta}=\mathbb{F}^{\beta \alpha}$. Thus we conclude from the above equation that $\mathbb{F}$ is identically zero: $\mathbb{F}^{\alpha \beta}=0$.

The third equation from eq. (3.5) leads to the expression (3.3) for $\mathbb{B}$. Using the third equation from eq. (3.5) and the fact that $\rho$ 's anticommute, the first equation from eq. (3.6) can written as in eq. (3.4).

In the supersymmetric case a closed expression for the star-product can be derived from the path integral. It reads

$$
\begin{aligned}
f \star g(x, \theta)= & f(x, \theta) \exp \left(\frac{-i \hbar}{2} \overleftarrow{\partial}_{\alpha} \mathbb{C}^{\alpha i} \vec{\partial}_{i}\right) \exp \left(\frac{i \hbar}{2} \overleftarrow{\partial}_{i} \mathbb{B}^{i j} \vec{\partial}_{j}\right) \exp \left(\frac{i \hbar}{2} \overleftarrow{\partial}_{i} \mathbb{C}^{\alpha i} \vec{\partial}_{\alpha}\right) \times \\
& \times \exp \left(\frac{\hbar^{2}}{12} \mathbb{C}^{\alpha k} \partial_{\alpha} \mathbb{B}^{i j}\left(\overleftarrow{\partial}_{i} \vec{\partial}_{j} \vec{\partial}_{k}-\overleftarrow{\partial}_{i} \overleftarrow{\partial}_{k} \vec{\partial}_{j}\right)\right) g(x, \theta)
\end{aligned}
$$

The Grassmann derivatives $\vec{\partial}_{\alpha}$ and $\overleftarrow{\partial}_{\alpha}$ in this equation act on the Grassmann variables as follows:

$$
\vec{\partial}_{1} \theta^{2} \theta^{1}=-\theta^{2}, \quad \vec{\partial}_{1} \theta^{1} \theta^{2}=\theta^{2}, \quad \theta^{1} \theta^{2} \overleftarrow{\partial}_{1}=-\theta^{2}, \quad \theta^{2} \theta^{1} \overleftarrow{\partial}_{1}=\theta^{2} .
$$

We sketch the path integral derivation of eq. (3.10). Schematically, we can write the formula (2.29) as

$$
\langle f(X, \Theta) \exp (\mathbb{B}+\mathbb{C}) g(X, \Theta)\rangle=\sum_{m, n} \frac{1}{m !} \frac{1}{n !}\left\langle f(X, \Theta) \mathbb{B}^{m} \mathbb{C}^{n} g(X, \Theta)\right\rangle,
$$

where $\mathbb{B}=\mathbb{B}^{i j} \boldsymbol{\rho}_{i} \boldsymbol{\rho}_{j}$ and $\mathbb{C}=\mathbb{C}^{\alpha i} \boldsymbol{\xi}_{\alpha} \boldsymbol{\rho}_{i}$. Since $\mathbb{B}^{i j}$ and $\mathbb{C}^{\alpha i}$ are $x$-independent, the $\boldsymbol{\rho}$ 's in $\mathbb{B}$ from eq. (3.12) can Wick-contract only with the functions $f$ and $g$. The Wick contraction of $f$ and $\boldsymbol{\rho}_{i}$ gives a derivative of $f: \partial_{i} f$. Furthermore, if one of the $\boldsymbol{\rho}$ 's from $\mathbb{B}$ contracts with $f$, then the remaining $\boldsymbol{\rho}$ has to contract with $g$ to produce a non-vanishing result. This is because $\mathbb{B}^{i j}$ is anti-symmetric and so $\mathbb{B}^{i j} \partial_{i} \partial_{j} f$ vanishes. We will shortly show that this type of contraction gives rise to the second exponential factor in eq. (3.10). 
The fate of $\boldsymbol{\xi}_{\alpha}$ and $\boldsymbol{\rho}_{i}$ in $\mathbb{C}$ can also be determined: $\boldsymbol{\rho}$ contracts with $f$ and $\boldsymbol{\xi}$ contracts with $g$, and vice versa. This type of contraction gives rise to the first and third exponential factors in eq. (3.10). Note that if $\boldsymbol{\xi}$ and $\boldsymbol{\rho}$ contract with the same function, then the corresponding contribution vanishes since the resulting Feynman integral vanishes:

$$
\int d \phi(a, z) \wedge d \phi(a, z)=0
$$

where $a$ is a point on the real-axis, by the anticommutativity of the wedge product.

Since $\mathbb{B}^{i j}$ is linear in $\Theta$, it can also contract with $\boldsymbol{\xi}_{\alpha}$ in $\mathbb{C}$. The three $\boldsymbol{\rho}$ 's from $\mathbb{B}$ and $\mathbb{C}$ have to contract with the functions $f$ and $g$. This type of contraction gives rise to the last exponential factor in eq. (3.10).

We now do some combinatorics to show that three different types of contractions just described indeed exponentiate into the formula (3.10). Let us pick $k \mathbb{B}$ 's and $k \mathbb{C}$ 's from eq. (3.12). There are $m ! /(k !(m-k) !)$ times $n ! /(k !(n-k) !)$ ways of doing that. There are $k$ ! ways of contracting $k \mathbb{B}$ 's with $k \mathbb{C}$ 's. Combining these combinatorial factors with the factorials from eq. 3.12 we have

$$
\frac{\mathbb{B}^{m-k}}{(m-k) !} \frac{\mathbb{C}^{n-k}}{(n-k) !} \frac{(\mathbb{C} \partial \mathbb{B})^{k}}{k !} .
$$

The fact that the Grassmann partial derivatives in eq. (3.10) act as in eq. (3.11) comes from the following observation. Consider the correlator

$$
\left\langle\cdots \Theta \Theta \cdots \Theta\left(\boldsymbol{\xi}_{\alpha} \mathbb{C}^{\alpha i} \boldsymbol{\rho}_{i}\right) \cdots\right\rangle
$$

where the $\boldsymbol{\Theta}$ 's came from the Taylor expansion of $f(\theta+\boldsymbol{\Theta})$. In order to Wick-contract a $\boldsymbol{\Theta}$ with $\boldsymbol{\xi}$, we have to bring it next to the $\boldsymbol{\xi}$. Thus the Wick-contraction with the $\boldsymbol{\xi}_{\alpha}$ is effectively equivalent to taking the derivative $\overleftarrow{\partial}_{\alpha}$.

The numerical coefficients in the exponents in eq. (3.10) can be found using the integrals [4]

$$
\int d \phi\left(z_{1}, 0\right) \wedge d \phi\left(z_{1}, 1\right)=2 \pi^{2}
$$

and

$$
\int d \phi\left(z_{1}, 0\right) \wedge d \phi\left(z_{1}, 1\right) \wedge d \phi\left(z_{2}, z_{1}\right) \wedge d \phi\left(z_{2}, 1\right)=\frac{4 \pi^{4}}{3} .
$$

Each of the correlators (2.33) which appear as the coefficients of $\mathbb{B}^{i j} \partial_{i} f \partial_{j} g, \mathbb{C}^{\alpha i} \partial_{\alpha} f \partial_{i} g$ and $\mathbb{C}^{\alpha i} \partial_{i} f \partial_{\alpha} g$ is proportional to the integral (3.16). The correlator which appears as the coefficient of $\mathbb{C}^{\alpha k} \partial_{k} \mathbb{B}^{i j} \partial_{i} f \partial_{j} \partial_{\alpha} g$ is proportional to the integral (3.17). The signs in the exponents in eq. (3.10) can be determined from the path integral as well.

Eq. (3.10) can, alternatively, be derived directly from the algebra (1.1) using the BakerHausdorff formula:

$$
\mathrm{e}^{A} \mathrm{e}^{B}=\mathrm{e}^{A+B+\frac{1}{2}[A, B]+\frac{1}{12}([A,[A, B]]+[[A, B], B])+\cdots} .
$$


Thinking of the star-product as a matrix product and using the Baker-Hausdorff formula, we find:

$$
\begin{aligned}
\mathrm{e}^{i(p \cdot x+\eta \cdot \theta)} \star \mathrm{e}^{i(\tilde{p} \cdot x+\tilde{\eta} \cdot \theta)}=\exp ( & i(p+\tilde{p}) \cdot x+i(\eta+\tilde{\eta}) \cdot \theta+\frac{i \hbar}{2}\left(\eta_{\alpha} \mathbb{C}^{\alpha i} \tilde{p}_{i}+p_{i} \mathbb{C}^{\alpha i} \tilde{\eta}_{\alpha}\right)- \\
& \left.-\frac{i \hbar}{2} p_{i} \tilde{p}_{j} \mathbb{B}^{i j}-\frac{i \hbar^{2}}{12} \mathbb{C}^{\alpha k} \partial_{\alpha} \mathbb{B}^{i j}\left(p_{i} \tilde{p}_{j} \tilde{p}_{k}-p_{i} p_{k} \tilde{p}_{j}\right)\right) \cdot
\end{aligned}
$$

Since

$$
\begin{aligned}
\mathrm{e}^{i p \cdot x} \overleftarrow{\partial}_{i}=i p_{i} \mathrm{e}^{i p \cdot x}, & \vec{\partial}_{i} \mathrm{e}^{i \tilde{p} \cdot x}=i \tilde{p}_{i} \mathrm{e}^{i \tilde{p} \cdot x} \\
\mathrm{e}^{i \eta \cdot \theta} \overleftarrow{⿱}_{\alpha}=i \eta_{\alpha} \mathrm{e}^{i \eta \cdot \theta}, & \vec{\partial}_{\alpha} \mathrm{e}^{i \tilde{\eta} \cdot \theta}=-i \tilde{\eta}_{\alpha} \mathrm{e}^{i \tilde{\eta} \cdot \theta}
\end{aligned}
$$

the quadratic and cubic terms in momenta in the exponent in eq. (3.19) lead, after the substitutions

$$
\begin{array}{ll}
p_{k} \rightarrow-i \overleftarrow{\partial}_{k}, & \tilde{p}_{k} \rightarrow-i \vec{\partial}_{k} \\
\eta_{\alpha} \rightarrow-i \overleftarrow{\partial}_{\alpha}, & \tilde{\eta}_{\alpha} \rightarrow i \vec{\partial}_{\alpha}
\end{array}
$$

to the star-product eq. (3.10).

\section{Summary}

Let us briefly summarize our results:

- A path-integral formula (2.1) for the associative star-product of functions of bosonic and fermionic coordinates $\left(x^{i}, \theta^{\alpha}\right)$ is proposed. $\theta$ is taken to be a Majorana spinor in $n$ spacetime dimensions.

- The BV action $S(2.6)$ of the two-dimensional field theory depends on the background fields $\mathbb{B}, \mathbb{F}$ and $\mathbb{C}$ from eq. (1.2). From the classical master equation $(S, S)_{\mathrm{BV}}=0$, we derived the associativity conditions $(2.26)$ for the background fields.

- An operational formula (2.29) is given for the star-product of functions of $x$ and $\theta$ for general $x$ - and $\theta$-dependent background fields.

- The condition of the supersymmetry invariance of the action (2.6) turned out to be very restrictive. The supersymmetric backgrounds are given by the equations (3.2), (3.3) and (3.4). The supersymmetry transformations (3.1) are $N=1$ or $N=2$ depending on the dimension $n$ of the spacetime. An explicit formula (3.10) for the supersymmetric star-product is derived from the path-integral.

Ref. [7] gives a deformation of $N=2, d=4$ superspace using symplectic-Majorana spinors. It turns out that a non-vanishing anticommutator

$$
\{\theta, \theta\} \neq 0
$$

is compatible with the $N=2$ supersymmetry. With a minor modification of our action (2.6) the case of the non-Majorana spinors can be dealt with. 


\section{Acknowledgments}

The work of I.C. is supported by a Sherman Fairchild Prize Fellowship. The work of C.C. is supported in part by the DOE Grant DE-FG03-92-ER40701.

\section{References}

[1] F. Bayen, M. Flato, C. Fronsdal, A. Lichnerowicz and D. Sternheimer, Deformation theory and quantization I Ann. Phys. (NY) 11 (1978) 61; Deformation theory and quantization II Ann. Phys. (NY) 11 (1978) 111.

[2] M. De Wilde and P.B.A. Lecomte, Existence of star-products and of formal deformations in Poisson Lie algebra of arbitrary symplectic manifolds, Lett. Math. Phys. 7 (1983) 487.

[3] B. Fedosov, A simple geometric construction of deformation quantization, J. Diff. Geom. 40 (1994) 2; J. Diff. Geom. 40 (1994) 213.

[4] M. Kontsevich, Deformation quantization of poisson manifolds, I, q-alg/9709040.

[5] A.S. Cattaneo and G. Felder, A path integral approach to the Kontsevich quantization formula, Commun. Math. Phys. 212 (2000) 591, math.QA/9902090.

[6] F.A. Berezin, Feynman path integrals in a phase space Sov. Phys. Usp. 23 (1981) 763;

I.A. Batalin and E.S. Fradkin, Operator quantization and abelization of dynamical systems subject to first class constraints, Riv. Nuovo Cim. 9 (1986) 1;

E. Fradkin and V. Linetsky, Quantization and cocycles on the supertorus and large- $N$ limits for the classical lie superalgebras, Mod. Phys. Lett. A 6 (1991) 217.

E. Gozzi and M. Reuter, Quantum deformed geometry on phase space, Mod. Phys. Lett. A 8 (1993) 1433 .

I.V. Tyutin, The general form of the star-product on the grassman algebra, Theor. Math. Phys. 127 (2001) 619-631 hep-th/0101046.

[7] D. Klemm, S. Penati and L. Tamassia, Non(anti)commutative superspace, Class. and Quant. Grav. 20 (2003) 2905 hep-th/0104190.

[8] P. Kosinski, J. Lukierski, P. Maslanka and J. Sobczyk, Quantum deformation of the Poincaré supergroup and $\kappa$ deformed superspace, J. Phys. A 27 (1994) 6827 hep-th/9405076.

[9] N. Berkovits, Covariant quantization of the Green-Schwarz superstring in a Calabi-Yau background, Nucl. Phys. B 431 (1994) 258 hep-th/9404162.

[10] H. Ooguri and C. Vafa, The c-deformation of gluino and non-planar diagrams, hep-th/0302109.

[11] L. Cornalba, M.S. Costa and R. Schiappa, D-brane dynamics in constant Ramond-Ramond potentials and noncommutative geometry, hep-th/0209164.

[12] J. de Boer, P.A. Grassi and P. van Nieuwenhuizen, Non-commutative superspace from string theory, hep-th/0302078.

[13] P.A. Grassi, G. Policastro and P. van Nieuwenhuizen, The covariant quantum superstring and superparticle from their classical actions, Phys. Lett. B 553 (2003) 96 hep-th/0209026.

[14] I.A. Batalin and G.A. Vilkovisky, Gauge algebra and quantization, Phys. Lett. B 102 (1981) 27; Quantization of gauge theories with linearly dependent generators, Phys. Rev. D 28 (1983) 2567 . 
[15] M. Alexandrov, M. Kontsevich, A. Schwartz and O. Zaboronsky, The geometry of the master equation and topological quantum field theory, Int. J. Mod. Phys. A 12 (1997) 1405 hep-th/9502010.

[16] J.-S. Park, Topological open p-branes, hep-th/0012141.

[17] A.S. Cattaneo and G. Felder, On the AKSZ formulation of the Poisson sigma model, Lett. Math. Phys. 56 (2001) 163, nath.QA/0102108. 